\title{
Impact of chromatin condensation assessment by aniline blue test on seminal parameters and outcomes in egg donation program
}

\author{
Filomena Scarselli $^{1 *}$, Giulia Pirastu ${ }^{1}$, Erika Iovine ${ }^{1}$, Simona Gatti $^{1}$, Saverio Muzzì ${ }^{1}$, Pierfrancesco Greco ${ }^{1}$, Maria Giulia Minasi ${ }^{1}$ and Ermanno \\ Greco $^{1,2}$ \\ ${ }^{1}$ Centre for Reproductive Medicine, Villa Mafalda, Monte delle Gioie, 00199 Rome, Italy \\ ${ }^{2}$ Saint Camillus International University of Health and Medical Sciences (UniCamillus), via di Sant'Alessandro, 00131 Rome, Italy
}

\begin{abstract}
Objective: In the present study, we aimed to evaluate sperm DNA chromatin integrity with the aniline blue staining (AB) and its possible impact on sperm parameters. We stabilized a predictive $\mathrm{AB}$ cut-off value for semen count and motility and analyzed the effect of chromatin decondensation in oligoastenozoospermic patients in oocytes donation (OD) program.

Methods:172 couples who underwent OD cycles between 2016 and 2018 were retrospectively included. The AB test was performed on total semen and post swim-up samples. Multiple Linear regression was used to check the correlation between the $\mathrm{AB}$ test and male characteristics (BMI and age) and semen parameters (morphology, concentration and motility). This association was further confirmed by ROC analysis that revealed a predictive AB test cut-off value. Finally, to verify if sperm quality influence clinical outcomes, we compared two subgroups with a positive $A B$ test divided in oligoastenozoospermic $(n=12)$ and normozoospermic $(\mathrm{n}=58)$.

Results: The distribution of $\mathrm{AB}$ staining was similar in both total and swim-up samples of 172 men $(p=0,216)$. AB test negative correlated with sperm motility $(p=0,008)$ and concentration $(p=0,001)$, but not with morphology $(p=0,6)$. On ROC analysis, sperm chromatin maturity revealed a $19 \%$ cut-off value of $\mathrm{AB}$ test for normal semen concentration (AUC $=0.675$, IC 95\%: $0.708-0.622, \mathrm{p}<0.05)$ and normal motility (AUC $=0.665$, IC $95 \%$ : $0.667-0.649$, $\mathrm{p}<0.05$ ). Comparison between subgroups showed a significant decrease in pregnancy positive tests $(p=0,03)$ and live birth rates $(\mathrm{LBR})(p=0,040)$ in oligoasthenozoospermic with $\mathrm{AB}$ test $\geq 19 \%$.

Conclusions: The present study detected a possible key role of sperm chromatin integrity in sperm parameters and clinical outcomes in OD program. An increase of the sample size is needed in order to confirm our findings.
\end{abstract}

\section{Introduction}

Female factor has always been considered crucial for achieving pregnancy in Medically Assisted Reproduction (MAR). Advanced maternal age (AMA) has a negative impact on in vitro fertilization (IVF) results: it affects oocytes and, consequently, embryo quality, by increasing chromosomal aneuploidies [1-3]. However, in the last years, several studies have focused their attention on the involvement of paternal factor in Assisted reproduction techniques (ART) outcomes [4-6]. It is known that the male factor causes infertility in about $50 \%$ of infertile couples [7] and it is estimated that about $15 \%$ of men with normal semen parameters have problems in achieving pregnancy [8]. Paternal factor relates to a reduction in fertilization rates and embryo development [9-11]. In many cases, routine semen analysis (spermiogram) is the only criterion for the selection of spermatozoa during IVF, a condition that has been extensively criticized. In addition, other authors concluded that men with normal semen parameters may still be infertile [12-14]. Recently, scientific attention has focused on other quality markers such as the structural and functional integrity of sperm and its decisive role in ART success [15-17]. In fact, sperm quality could be assessed not only considering standard semen parameters, but also sperm DNA damage, sperm membrane permeability and sperm chromatin condensation $[18,19]$. Sperm nuclear integrity is guaranteed by a correct chromatin packaging, which is normally assured by the replacement of histones with protamines (protamination) to form tight
DNA-proteins structure [20]. It has been evaluated that incorrect protamination is associated with advanced paternal age (APA) and can alter both sperm fertilization capacity and early embryo development [21]. Therefore, sperm chromatin condensation may have an important role in influencing sperm competence to achieve pregnancy in ART [22-25]. In the present study, we evaluate sperm DNA chromatin integrity with Aniline blue $(\mathrm{AB})$ staining test that distinguish unstained mature spermatozoa from stained immature ones [26-27]. The aim of the study is to define a predictive $\mathrm{AB}$ cut-off value for semen parameters and to analyze the impact of chromatin decondensation in oligoastenozoospermic patients in OD cycles.

\section{Materials and methods}

\section{Study population}

We retrospectively analyzed 172 male patients in OD model enrolled in European Hospital Fertility Center between January 2016

${ }^{\star}$ Correspondence to: Filomena Scarselli, MSc, Centre for Reproductive Medicine, Villa Mafalda, Italy

Key words: aniline blue, egg donation, sperm parameters

Received: June 16, 2021; Accepted: July 05, 2021; Published: July 12, 2021 
and December 2018. Inclusion criteria were: history of infertility of 1 year or longer, normal or sub-fertile seminal parameters, cycles with 8-9 vitrified oocytes with a post-warming survival rate $>50 \%$. Only fresh ejaculate semen was included. Patients with azoospermia, hypogonadism, testicular torsion, concomitant urogenital infections, previous chemotherapy or radiotherapy and drug therapy were excluded from the analysis. All patients were free from type- $1 / 2$ human immunodeficiency virus (HIV), hepatitis B virus, hepatitis C virus, Cytomegalovirus, Treponema pallidum and Syphilis. Our population was evaluated for $A B$ test which was performed both on semen sample and after swim-up treatment. 70 patients of 172 with the positive $A B$ test cut-off, detected in this study, were considered and divided into two subgroups: oligoasthenozoospermic and normozoospermic. The study was conducted in accordance with the principles expressed in the Declaration of Helsinki and an informed consent was obtained from the couples with the approval of the internal ethics commission of the European Hospital.

\section{Semen assessment and preparation}

On the day of insemination, all subjects underwent semen analysis: fresh semen was collected in a sterile jar by masturbation after 3-5 days of sexual abstinence. Semen analysis has been performed following the WHO 2010 [28] at $5^{\circ}$ percentile: semen volume, $1,5(1,4-1,7) \mathrm{ml}$; total sperm number, 39 (33-46) million per ejaculate, sperm concentration, 15 (12-16) million per ml; progressive motility, 32\% (31-34); total (progressive, non- progressive) motility, 40\% (38-42); morphology $4,0 \%(3,0-4,0)$. Swim-up was performed for semen preparation. The sperm count, motility and morphology (Diff Quick staining, MBT Medical Biological Technologies, Italy) before and after swim-up, were analyzed according to WHO 2010 guidelines [28].

\section{Aniline Blue staining}

$\mathrm{AB}$ test was performed both on native semen and swimp-up. An aliquot of the samples was smeared on slides and let them dried on air. After that, air-dried smears were fixed for $15 \mathrm{~min}$ in $37 \%$ formaldehyde in phosphate buffered saline solution $(\mathrm{pH} \mathrm{7.2)}$ and stained with $5 \%$ $(\mathrm{w} / \mathrm{v})$ aqueous AB (Sigma ${ }^{\oplus}$, Germany) in $4 \%$ acetic acid ( $\left.\mathrm{pH} 3.5\right)$ for 2 min [29]. The slides were washed with sterile water and spermatozoa observed for $\mathrm{AB}$ staining: they were positive if the dark blue dye occupied the entire region of the sperm head or the post-acrosomal region; conversely, they were considered negative if the sperm heads were colorless.

\section{Laboratory and Clinical procedures}

Donated oocytes were warmed following the Kitazato procedure (Kitazato thawing Kit; BioPharma, Shizouka, Japan). One and a half hour after warming, surviving oocytes underwent ICSI and only correctly fertilized (2PN) oocytes were analyzed. Embryo morphology was assessed using our routine scoring system [30] and blastocysts morphology was evaluated with the scoring system described by Gardner and Schoolcraft [31]. Embryo culture was performed in incubators at $37^{\circ} \mathrm{C}, 5 \% \mathrm{O} 2$ and $6 \% \mathrm{CO} 2$ in sequential media (Quinn's Advantage Medium; SAGE, USA). In OD cycles, our laboratory policy is to transfer 1 or 2 embryos on day-3 or 1 blastocyst on day-5. Embryo transfer was performed under transabdominal ultrasound guidance using a Wallace catheter (Wallace; Smith Medical, Dublin, Ireland) [32]. Supernumerary embryos were vitrified (Kitazato vitrification Kit; BioPharma, Shizouka, Japan). The $\beta$-HCG value was assessed 14 days after embryo transfer. Clinical pregnancy was confirmed by ultrasound visualization of the gestational sac/s with fetal heartbeat.

\section{Statistical analysis}

Male age, body mass index (BMI) and semen parameters are presented as median value (min-max). Normal distribution of $A B$ test was tested by the Kolmogorov-Smirnov test and to evaluate if there were any difference in the results, the nonparametric Mann-Whitney $U$ test was utilized. Multiple Linear regression was used to check the correlation between the $\mathrm{AB}$ test, $\mathrm{BMI}$, male age, morphology and semen concentration or motility. The predictive $A B$ test cut-off of standard semen parameters was verified using the ROC curve and the area under the curve (AUC), considering confidence interval (CI 95\%). We evaluated differences between subgroups in terms of clinical outcomes using Wilcoxon test. The Statistical Package for Social Sciences (SPSS 23.0, Chicago, IL, USA) was used. $p$ value $\leq .05$ was considered as significant.

\section{Results}

Male population of 172 OD cycles was described in table 1 by reporting age, BMI, semen parameters and $\mathrm{AB}$ values before and after swim-up. The male median (min-max) age was $44,76(36.00-53.92)$ years old; male median (min-max) BMI was 28,70 (27.00-34.00). The distribution of $\mathrm{AB}$ test values was similar in the whole sample and in the swim-up sample $(p=0,262)$ (Figure 1). As shown in table 2, there was a negative correlation between $\mathrm{AB}$ test value and sperm concentration $(p=0,001)$ and motility $(p=0,008)$; whereas there wasn't any correlation with morphology $(p=0,6)$. Male characteristics such as male age $(p=0,3)$ and BMI $(p=0,07)$ did not relate to sperm nuclear integrity. Subsequently, the 172 male patients were divided considering each single sperm parameter at a time: 148 had a normal concentration and 24 abnormal; 154 had a normal total motility and 18 abnormal; 75 had a normal morphology and 97 abnormal.

As showed from ROC curve, sperm chromatin maturity did not correlate with morphology (AUC $=0.550$, IC 95\%: 0.103-0.87, $\mathrm{p}=0.245$ ) (Figure 2a) but revealed a significant predictive value of $\mathrm{AB}$ test for normal semen concentration (AUC=0.675, IC 95\%: 0.708 $0.622, \mathrm{p}<0.05$, cut off $=19 \%$ ) (Figure $2 \mathrm{~b}$ ) and for normal motility (AUC=0.665, IC 95\%: $0.667-0.649, \mathrm{p}<0.05$, cut-off=19\%) (Figure 2c). To verify if sperm quality influence clinical outcomes in OD cycles we compared two subgroups with positive $\mathrm{AB}$ test $(\geq 19 \%)$ divided in oligoastenozoospermic and normozoospermic. As showed in table 3 , there was a statistical significance difference in the ability to achieve pregnancy ( $B$-hCG positive test) $(p=0,03)$ and in the live birth rate (LBR) $(p=0,04)$ in the group with normal seminal parameters but with high $\mathrm{AB}$ cut-off $(\geq 19 \%)$.

\section{Discussion}

Our study used OD cycles as a model to better understand male impact and focused the attention on semen parameters, age, BMI and

Table 1. Values are expressed as median (R); R, range (min-max); BMI, body mass index $\mathrm{AB}$, aniline blue

\begin{tabular}{|l|c|}
\hline Principal charateristics of the $\mathbf{1 7 2}$ male partner in OD model \\
\hline Characteristics & Values \\
\hline Male age (years) & $24,67(36,00-53,92)$ \\
\hline Male BMI (kg/m ) & $28,70(27,00-34,00)$ \\
\hline Semen volume (ml) & $2,80(1,00-7,40)$ \\
\hline Sperm concentration (X10 /ml) & $39,00(5-80)$ \\
\hline Total sperm motility A+B+C (\%) & $58(15-95)$ \\
\hline Normal Sperm morphology (\%) & $3,0(1-6)$ \\
\hline Sperm concentration after swim-up (X10 /ml) & $20(0,80-95)$ \\
\hline Total sperm motility after Swim-up (\%) & $95,0(43-99)$ \\
\hline AB staining spermatozoa (\%) & $16,5(2-77)$ \\
\hline AB staining spermatozoa after swim-up (\%) & $12,5(2-61)$ \\
\hline
\end{tabular}




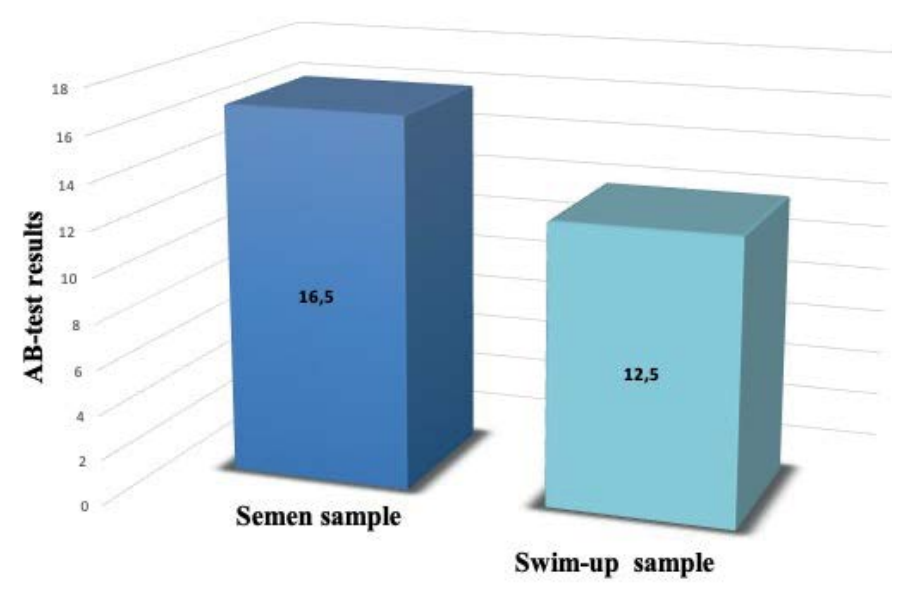

Figure 1. Comparison of $A B$ test results before and after swim-up. $A B$ test results are expressed as median value, nonparametric Mann-Whitney U test was utilized $(\mathrm{p}=0,262)$. $\mathrm{P}$-value $<0,05$ was considered to be significant

Table 2. BMI, body mass index; $\mathrm{AB}$, aniline blue. $P$-value $<0,05$ was considered to be significant

\begin{tabular}{|l|c|c|}
\hline $\begin{array}{l}\text { Multiple regression analysis. Correletion between sperm parameters, BMI, male age } \\
\text { with Aniline blue (AB)- test }\end{array}$ & P-value \\
\hline Male Parameters & Correlation coefficient $(\boldsymbol{r})$ & \\
\hline AB & & $\mathbf{0 , 0 0 1}$ \\
\hline Sperm concentration & $-0,14$ & $\mathbf{0 , 0 0 8}$ \\
\hline Sperm motility & $-0,21$ & 0,6 \\
\hline Sperm morfology & 0,26 & 0,07 \\
\hline Male BMI & 0,31 & 0,3 \\
\hline Male Age & 0.58 & \\
\hline
\end{tabular}

chromatin condensation evaluated by $\mathrm{AB}$ test. For sperm preparation was used the swim-up because it has been reported that the final spermatozoa have a better chromatin condensation compared to the density gradient [34]. We did not found a significant difference in $\mathrm{AB}$ values in the whole semen sample and after swim-up. In the whole semen sample, being the spermatogenesis a continuous process, it is possible to have different amounts of immature, mature and senescent spermatozoa with chromatin impairing condensation depending on day of abstinence [33-35]. It is reasonable to hypothesize that the swimup, which is a selection technique based on sperm motility, does not eliminate spermatozoa with DNA damage due to impaired chromatin condensation [36]. Multiple regression analysis between $A B$ values and male parameters did not show an association between $\mathrm{AB}$ with male age and BMI and sperm morphology; however, there was a negative correlation of chromatin maturity with concentration and motility $(\mathrm{p}<0.001)$ in agreement with other studies present in literature [27,3739]. As concern morphology, Pourmasumi et al. (2019) showed a negative correlation between this parameter and $A B$ staining [27], in contrast with our article where no correlation was found. Hammadeh et al. (2001) [40] suggested no correlation between chromatin condensation and sperm morphology, even if the authors declared that sperm chromatin assessment constitutes a valuable parameter in the male fertility, completely independent of conventional semen parameters.

We straightened this multiple regression analysis results by ROC curve that permitted us to find an $\mathrm{AB}$ test cut-off of $19 \%$ predictive for sperm count and motility, but not for morphology. Whereas several authors found an $\mathrm{AB}$ test cut-off in relation with clinical results $[26,41]$, we identified a cut-off value in relation to semen parameters that could be a potential index of male infertility. $\mathrm{AB}$ test $\geq 19 \%$ were more associated with men with abnormal concentration and motility (oligoasthenozoospermic) and this could be due to a different spermatogenic remodeling of chromatin resulting in greater histone retention $(>15 \%)$ or chromatin non-condensation $[38,42,43]$. To evaluate the influence of $\mathrm{AB}$ test cut-off of $19 \%$ on clinical outcomes, we considered OD cycles in which female factor (maternal age) has a heavy weight on the results [44]. In our study, oligoasthenozoospermic patients compared to normozoospermic, sperm chromatin decondensation did not affect ICSI results (oocyte fertilization and embryo development rates) but a decrease in pregnancy positive tests and LBR was detected. Our data could agree with other works that did not show an association between correct chromatin packaging and ICSI results [26]. However, it is known that alterations in DNA packaging can be associated [45] with semen parameters and ICSI results [21]. The impact of chromatin condensation on fertilization and embryo development rates is controversial, while it seems to have more influence on ART clinical outcomes as could be seen from our results [22,46-52]. Our
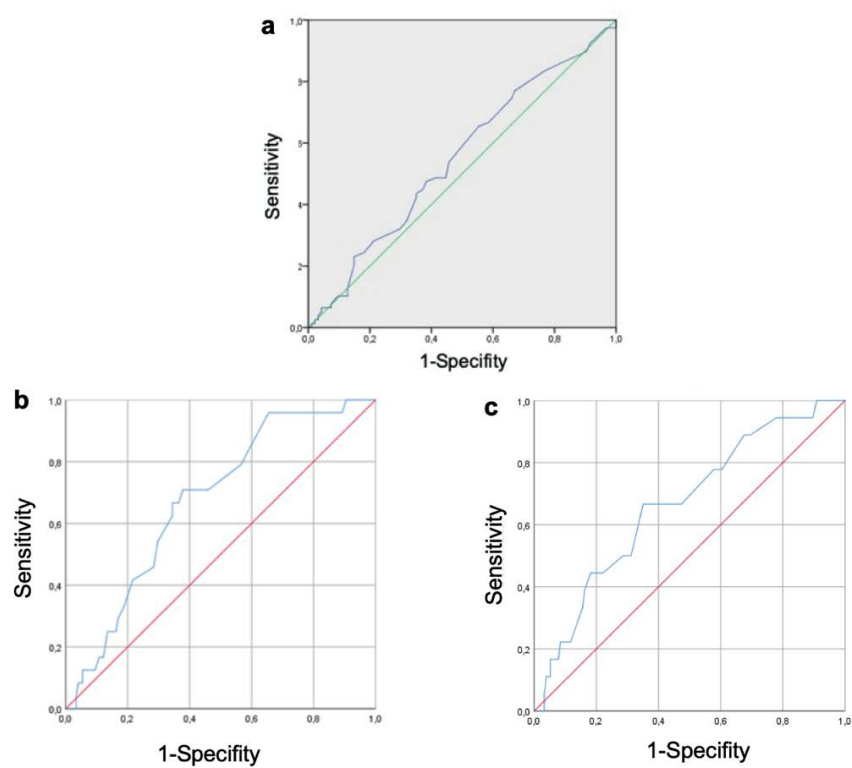

Figure 2. ROC CURVE. 2a) Receiving operating characteristic (ROC) curve of $\mathrm{AB}$ test for predicting semen morphology: $\mathrm{AUC}=0,550, p=0,245$, IC 95\%: $0,103-0,87$, cutoff point $=29 \%$. 2b) ROC curve of AB-test for predicting concentration: $A U C=0,675$, $p<0,05$, IC 95\%: $0.708-0.622$ cut-off point $=19 \%$. 2c) ROC curve of AB-test for predicting motility: $\mathrm{AUC}=0,665, p<0,05$ IC $95 \%$ : $0.667-0.649$ cut-off point $=19 \% . P$-value $<0,05$ was considered to be significant

Table 3. Population was divided according to $\mathrm{AB}$ test cut-off positive value (AB test $\geq$ $19 \%$ ). and semen total motility and concentration in 2 subgroups: oligoasthenozoospermic and normozoospermic

Comparison of ICSI outcomes between sub-groups with an AB test cut-off $\geq 19 \%$ in OD program.

\begin{tabular}{|l|c|c|c|}
\hline Variables & $\begin{array}{c}\text { Oligoasthenozoospermic } \\
(\mathbf{n = 1 2 )}\end{array}$ & $\begin{array}{c}\text { Normozoospermic } \\
(\mathbf{n = 5 8})\end{array}$ & P-value \\
\hline Oocyte survival rate (\%) & $76 / 89(85,39)$ & $1112 / 1267(87,77)$ & NS \\
\hline Fertilized oocytes (\%) & $52 / 76(68,42)$ & $842 / 1112(75,72)$ & NS \\
\hline Total Embryos (\%) & $41 / 52(78,85)$ & $641 / 842(76,13)$ & NS \\
\hline B-hCG positive test (\%) & $4 / 12(33,33)$ & $108 / 160(67,5)$ & $\mathbf{0 , 0 3}$ \\
\hline $\begin{array}{l}\text { Clinical pregnancies rate } \\
\text { (CPR\%) }\end{array}$ & $3 / 12(25,00)$ & $91 / 160(56,88)$ & 0,06 \\
\hline Live birth rate (LBR\%) & $3 / 12(25,00)$ & $95 / 160(59,38)$ & $\mathbf{0 , 0 4}$ \\
\hline Miscarriage (\%) & $0(0)$ & $12 / 105(11,43)$ & NS \\
\hline
\end{tabular}


data showed a correlation between a positive $\mathrm{AB}$ test and impaired sperm parameters: poorly packed sperm chromatin makes DNA more susceptible to reactive oxygen species (ROS) attack [16]. Oxidative stress condition has a negative effect both on mitochondrial functionality and DNA: this leads to a decrease in sperm motility and an impairment of DNA integrity [53]. In conclusion, sperm chromatin condensation is essential to assure a correct DNA packaging.

\section{Conclusion}

The present study evaluates the effect of chromatin integrity on seminal parameters of male partners in OD cycles. We found a predictive positive cut-off value of $19 \% \mathrm{AB}$ for sperm concentration and motility, but not for morphology. Our preliminary data focus on seminal parameters in relation to the method of the selection of spermatozoa with intact chromatin in men of advanced age undergoing OD cycles. A correct DNA packaging is essential for better success in ART techniques and our results could be an advice to pay more attention to patients with reduced motility (astenozoospermic).

\section{References}

1. Angell RR (1994) Aneuploidy in older women. Higher rates of aneuploidy in oocytes from older women. Hum Reprod 9: 1199-200. [Crossref]

2. Munné S, Alikani M, Tomkin G, Grifo J, Cohen J (1995) Embryo morphology, developmental rates, and maternal age are correlated with chromosome abnormalities. Fertil Steril 64: 382-391; Corrected and republished in 2019, 112(4 Supp11): e71-e80.

3. Wood C, Calderon I, Crombie A (1992) Age and fertility: results of assisted reproductive technology in women over 40 years. J Assist Reprod Genet 9: 482-484. [Crossref]

4. Lawson G, Fletcher R (2014) Delayed fatherhood. J Fam Plann Reprod Health Care 40: 283-288. [Crossref]

5. Cito G, Coccia ME, Picone R, Cocci A, Russo GI, Garaffa G, et al. (2019) Impact of advanced paternal age on the intracytoplasmic sperm injection (ICSI) outcomes in donor egg cycles. Transl Androl Urol 8: S22-S30. [Crossref]

6. Cocuzza M, Athayde KS, Agarwal A, Sharma R, Pagani R, et al. (2008) Age-related increase of reactive oxygen species in neat semen in healthy fertile men. Urology 271: 490-494.

7. Agarwal A, Mulgund A, Hamada A, Chyatte MR (2015) A unique view on male infertility around the globe. Reprod Biol Endocrinol 13-37. [Crossref]

8. Evgeni E, Charalabopoulos K, Asimakopoulos B (2014) Human sperm DNA fragmentation and its correlation with conventional semen parameters. J Reprod Infertil 15: 2-14. [Crossref]

9. Belloc S, Benkhalifa M, Junca AM, Dumont M, Bacrie PC, et al. (2009) Paternal age and sperm DNA decay: discrepancy between chromomycin and aniline blue staining. Reprod Biomed Online 19: 264-269. [Crossref]

10. Kidd SA, Eskenazi B, Wyrobek AJ (2001) Effects of male age on semen quality and fertility: a review of the literature. Fertil Steril 75: 237-248.

11. Winkle T, Rosenbusch B, Gagsteiger F, Paiss T, Zoller N (2009) The correlation between male age, sperm quality and sperm DNA fragmentation in 320 men attending a fertility center. J Assist Reprod Genet 26: 41-46. [Crossref]

12. Aydos OS, Yükselten Y, Kaplan F, Sunguroğlu A, Aydos K (2015) Analysis of the correlation between sperm DNA integrity and conventional semen parameters in infertile men. Turk J Urol 41: 191-197.

13. Bungum M, Bungum L, Giwercman A (2011) Sperm chromatin structure assay (SCSA): a tool in diagnosis and treatment of infertility. Asian J Androl 13: 69-75. [Crossref]

14. Omran HM, Bakhiet M, Dashti MG (2013) DNA integrity is a critical molecular indicator for the assessment of male infertility. Mol Med Rep 7: 1631-1635. [Crossref]

15. Lewis SE, Aitken RJ (2005) DNA damage to spermatozoa has impacts on fertilization and pregnancy. Cell Tissue Res 322: 33-41. [Crossref]

16. Pourmasumi S, Sabeti P, Rahiminia T, Mangoli E, Tabibnejad N, et al. (2017) The etiologies of DNA abnormalities in male infertility: An assessment and review. Int $J$ Reprod Biomed 15: 331-344. [Crossref]

17. Zini A, Boman JM, Belzile E, Ciampi A (2008) Sperm DNA damage is associated with an increased risk of pregnancy loss after IVF and ICSI: systematic review and metaanalysis. Hum Reprod 23: 2663-2668. [Crossref]
18. Agarwal A (2009) Textbook of assisted reproductive technologies. (3rd ed.), London, Informa Healthcare, 67-84.

19. Bieniek JM, Drabovich AP, Lo KC (2016) Seminal biomarkers for the evaluation of male infertility. Asian J Androl 18: 426-433. [Crossref]

20. Utsuno H, Miyamoto T, Oka K, Shiozawa T (2014) Morphological alterations in protamine-deficient spermatozoa. Hum Reprod 29: 2374-2381. [Crossref]

21. Mangoli E, Khalili MA, Talebi AR, Ghasemi-Esmailabad S, Hosseini A (2018) Is there any correlation between sperm parameters and chromatin quality with embryo morphokinetics in patients with male infertility? Andrologia 50: e12997. [Crossref]

22. Irez T, Sahmay S, Ocal P, Goymen A, Senol H, et al. (2015) Investigation of the association between the outcomes of sperm chromatin condensation and decondensation tests, and assisted reproduction techniques. Andrologia 47: 438-447.

23. Robinson L, Gallos ID, Conner SJ, Rajkhowa M, Miller D, et al. (2012) The effect of sperm DNA fragmentation on miscarriage rates: a systematic review and meta-analysis. Hum Reprod 27: 2908-2917. [Crossref]

24. Simon L, Murphy K, Shamsi MB, Liu L, Emery B, et al. (2014) Paternal influence of sperm DNA integrity on early embryonic development. Human Reprod 29: 2402-2412. [Crossref]

25. Talebi AR, Fesahat F, Mangoli E, Ghasemzadeh J, Nayeri M, et al. (2016) Relationship between sperm protamine deficiency and apoptosis in couples with unexplained repeated spontaneous abortions. Int J Reprod Biomed 14: 199-204. [Crossref]

26. Gill K, Rosiak A, Gaczarzewicz D, Jakubik J, Kurzawa, E, et al. (2018) The effect of human sperm chromatin maturity on ICSI outcomes. Human Cell 31(3): 220-231.

27. Pourmasumi S, Khoradmehr A, Rahiminia T, Sabeti P, Talebi AR, et al. (2019) Evaluation of sperm chromatin integrity using Aniline Blue and Toluidine Blue Staining in Infertile and normozoospermic men. J Reprod Infertil 20: 95-101.

28. World health organization: WHO Laboratory Manual for the Examination and Processing of Human Semen. (2010) 5th ed, Geneva, Switzerland, WHO Press.

29. Alkhayal A, San Gabriel M, Zeidan K, Alrabeeah K, Noel D, et al. (2013) Sperm DNA and chromatin integrity in semen samples used for intrauterine insemination. $J$ Assist Reprod Genet 30: 1519-1524. [Crossref]

30. Minasi MG, Colasante A, Riccio T, Ruberti A, Casciani V, et al. (2016) Correlation between aneuploidy, standard morphology evaluation and morphokinetic development in 1730 biopsied blastocysts: a consecutive case series study. Hum Reprod 31: 22452254. [Crossref]

31. Gardner DK, Schoolcraft WB (1999) Culture and transfer of human blastocysts. Curr Opin Obstet Gynecol 11: 307-311. [Crossref]

32. Litwicka K, Mencacci C, Arrivi C, Varricchio MT, Caragia A, et al. (2018) HCG administration after endogenous LH rise negatively influences pregnancy rate in modified natural cycle for frozen-thawed euploid blastocyst transfer: a pilot study. $J$ Assist Reprod Genet 35: 449-455. [Crossref]

33. Lestari SW, Lestari SH, Pujianto DA (2018) Sperm quality after swim up and density gradient centrifugation sperm preparation with supplementation of alpha lipoic acid (ALA): A preliminary study. AIP Conference Proceedings 1933, 030015.

34. Saylan A, Erimsah S (2019) High quality human sperm selection for IVF: A study on sperm chromatin condensation. Acta Histochem 121: 798-803. [Crossref]

35. Scarselli F, Cursio E, Muzzì S, Casciani V, Ruberti A, et al. (2019) How 1 h of abstinence improves sperm quality and increases embryo euploidy rate after PGT-A: a study on 106 sibling biopsied blastocysts. J Assist Reprod Genet 36: 1591-1597. [Crossref]

36. De Martin H, Miranda EP, Cocuzza MS, Monteleone PAA (2019) Density gradient centrifugation and swim-up for ICSI: useful, unsafe, or just unsuitable? J Assist Reprod Genet 36: 2421-2423. [Crossref]

37. Al-Fahham AA, Al-Sultani YK, Muhammad-Ali AK (2014) Using sperm chromatin staining techniques as a predictive diagnostic tool for male infertility. Kufa J Nurs Sci 4: 56-64.

38. Kazerooni T, Asadi N, Jadid L, Kazerooni M, Ghanadi A, et al. (2009) Evaluation of sperm's chromatin quality with acridine orange test, chromomycin A3 and aniline blue staining in couples with unexplained recurrent abortion. J Assist Reprod Genet 26(1112): 591-596. [Crossref]

39. Varghese AC, Bragais FM, Mukhopadhyay D, Kundu S, Pal M, et al. (2009) Human sperm DNA integrity in normal and abnormal semen samples and its correlation with sperm characteristics. Andrologia 41: 207-215. [Crossref]

40. Hammadeh M, Zeginiadov T, Rosenbaum P, Georg T, Schnidt W, et al. (2001) Predictive value of sperm chromatin condensation (aniline blue staining) in the assessment of male fertility. Arch Androl 46: 99-104. 
41. Irez T, Dayioglu N, Alagöz M, Karatas S, Güralp O (2018) The use of aniline blue chromatin condensation test on prediction of pregnancy in mild male factor and unexplained male infertility. Andrologia 50: e13111.

42. Asmarinah AS, Syauqy A, Umar LA, Lestari SW, Mansyur E, et al. (2016) Sperm chromatin maturity and integrity correlated to zygote development in ICSI program. Syst Biol Reprod Med 62: 309-316.

43. Talebi AR, Vahidi S, Aflatoonian A, Ghasemi N, Ghasemzadeh J, et al. (2012) Cytochemical evaluation of sperm chromatin and DNA integrity in couples with unexplained recurrent spontaneous abortions. Andrologia 44: 462-470. [Crossref]

44. Spandorfer S (2018) Egg donation model: an excellent way to isolate and analyze the impact of the male partner on assisted reproductive technology outcome. Fertil Steril 110: 844. [Crossref]

45. Agarwal A, Said TM (2003) Role of sperm chromatin abnormalities and DNA damage in male infertility. Hum Reprod Update 9: 331-345. [Crossref]

46. Bach PV, Schlegel PN (2016) Sperm DNA damage and its role in IVF and ICSI. Basic Clin Androl 26: 15. [Crossref]

47. Galotto C, Cambiasso MY, Julianelli VL, Valzacchi GJR, Rolando RN, et al. (2019) Human sperm decondensation in vitro is related to cleavage rate and embryo quality in IVF. J Assist Reprod Genet 36: 2345-2355. [Crossref]
48. Sadeghi MR, Hodjat M, Lakpour N, Arefi S, Amirjannati N, et al. (2009) Effects of sperm chromatin integrity on fertilization rate and embryo quality following intracytoplasmic sperm injection. Avicenna J Med Biotechnol 1: 173-180. [Crossref]

49. Simon L, Emery BR, Carrell DT (2017) Review: Diagnosis and impact of sperm DNA alterations in assisted reproduction. Best Pract Res Clin Obstet Gynaecol 44: 38-56. [Crossref]

50. Tarozzi N, Nadalini M, Stronati A, Bizzaro D, Dal Prato L, et al. (2009) Anomalies in sperm chromatin packaging: implications for assisted reproduction techniques. Reprod Biomed Online 18: 486-495. [Crossref]

51. Zhang Z, Zhu L, Jiang H, Chen H, Chen Y, et al. (2015) Sperm DNA fragmentation index and pregnancy outcome after IVF or ICSI: a meta-anlysis. J Assist Reprod Genet 32: 17-26. [Crossref]

52. Zheng WW, Song G, Wang QL, Liu SW, Zhu XL, et al. (2018) Sperm DNA damage has a negative effect on early embryonic development following in vitro fertilization. Asian J Androl 20: 75-79. [Crossref]

53. Barratt CL, Aitken RJ, Bjorndahl L, Carrell DT, De Boer P, et al. (2010) Sperm DNA: organization, protection and vulnerability: from basic science to clinical applications a position report. Hum Reprod 25: 824-838. [Crossref]

Copyright: (2021 Scarselli F. This is an open-access article distributed under the terms of the Creative Commons Attribution License, which permits unrestricted use, distribution, and reproduction in any medium, provided the original author and source are credited. 